\title{
Rates and causes of stillbirth in a demographic surveillance site in Karachi, Pakistan
}

\author{
Muhammad Imran Nisar*, Muhammad Ilyas, Komal Naeem, Urooj Fatima, yasir Shafiq \\ and Fyezah Jehan
}

Pediatrics, Aga Khan University, Karachi, Pakistan

\section{Objective}

To determine burden, timing and causes of stillbirths in a prospective cohort of pregnant from a low income community setting in peri urban Karachi

\section{Introduction}

Stillbirth remained a neglected issue absent from mention in Millennium Development Goals. An estimated 2.6 million babies are stillborn every year withhighest rate in Pakistan, 43.1 stillbirths $/ 1000$ births. There is lack of good quality prospective population based data in Pakistanregarding burden, timing and causes of stillbirths

\section{Methods}

From Jan - Dec 2012, Community Helath Workers (CHWs) identified pregnant women through 3 monthly household visits.

Pregnant women were then followed up till end of their pregnancy. In case of a stillbirth, a detailed verbal autopsy (VA) interview was undertaken 2 weeks after the outcome by a research assistant. VA forms were then reviewed by 2 independent Physicians who assigned a cause for stillbirth. In case of disagreement, VA form was reviewed by a third physician. A consensus between two physicians was required for a definitive cause.

\section{Results}

There were a total of 273 stillbirths (3.04\%) reported. Stillbirth rate was $30.7 / 1000$ births. Distribution of antepartum and intrapartum stillbirths was $83 \%$ and $17 \%$. Three most common causes of stillbirths included pregnancy induced Hypertension(37\%), antepartum hemorrhage (10\%) and obstructed labor(6\%) (fig. 1).

\section{Conclusions}

We have reported a high burden of stillbirths that take place during the intrapartum period. This reemphasizes need for good quality antenatal care in these settings. Appropriate measure needs to be taken targeting most common causes of stillbirths, focusing on improved antepartum health care facilities
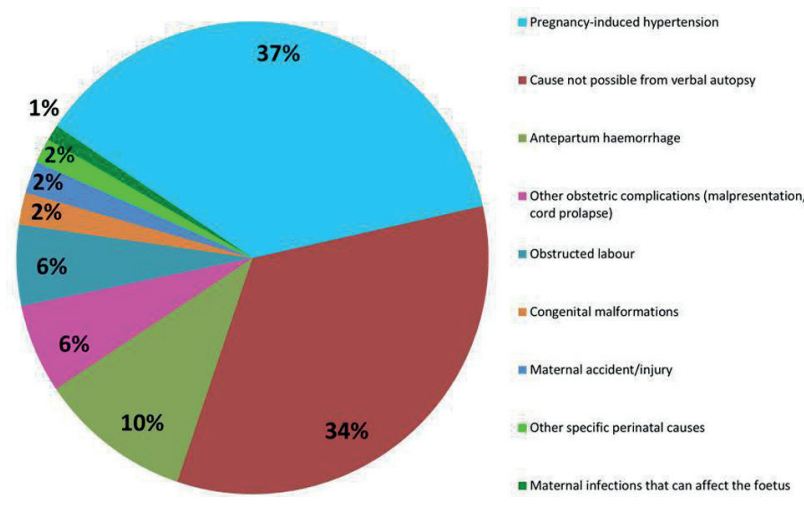

\section{*Muhammad Imran Nisar}

E-mail: imran.nisar@aku.edu

Keywords

Still birth; Burden; Low income countries 